\title{
Measurement of jet fragmentation using the ATLAS detector
}

\author{
Miguel Villaplana Perez ${ }^{* \dagger}$ \\ Department of Physics, University of Alberta, Edmonton AB, Canada \\ E-mail: Miguel.Villaplana.Perez@cern.ch
}

Gluon splitting to $b$-quark pairs is a unique probe of the properties of gluon fragmentation, as the identified $b$-tagged jets are related to the quark daughters of the initial gluon. We present a measurement of key differential distributions related to $g \rightarrow b \bar{b}$ using data collected with the ATLAS detector at $13 \mathrm{TeV}$. Track jets are used to probe angular scales below the standard $\mathrm{R}=0.4$ jet radius. The observables are unfolded to the particle level in order to facilitate direct comparison with predictions from simulations, and provide an important constraint to hadronization models. A measurement of the properties of jet fragmentation performed with proton-proton collision data collected with the ATLAS detector at $13 \mathrm{TeV}$ will also be presented. Charged particle tracks are used to measure charged particle multiplicity, the jet charge, the summed fragmentation function, the momentum transverse to the jet axis, and the radial profile of the jet. Each observable is unfolded to correct for acceptance and detector effects. Exclusive interpretations in terms of quarks and gluons are provided in order to directly compare with state-of-the-art calculations.

European Physical Society Conference on High Energy Physics - EPS-HEP2019 -

10-17 July, 2019

Ghent, Belgium

\footnotetext{
*Speaker.

${ }^{\dagger}$ On behalf of the ATLAS Collaboration.
} 


\section{Properties of $g \rightarrow b \bar{b}$ at small opening angles}

Gluon splitting to $b \bar{b}$ pairs at small opening angles is the main contribution to the background for searches with boosted Higgs bosons in the $H \rightarrow b \bar{b}$ channel [1]. The $g \rightarrow b \bar{b}$ process also contributes to many other important Standard Model (SM) measurements and searches by providing a source of additional real $b$-quark jets that can fake a signal for $b$-quarks originating from other processes (see e.g., Ref [2]). The modeling of $g \rightarrow b \bar{b}$ fragmentation provides a useful probe of quantum chromodynamics (QCD) and the large mass of the $b$-quark introduces a significant modification to the massless QCD splitting functions by screening the soft-emission singularity. The high transverse momentum and low angular separation regime for $g \rightarrow b \bar{b}$ is probed in the ATLAS experiment [3] using small-radius jets built from charged-particle tracks, used as proxies to $b$-quarks, within large-radius jets [4].

The kinematic properties of the $g \rightarrow b \bar{b}$ process are characterized by three quantities: the opening angle between the $b$-quarks $\Delta R(b, b)$; the momentum sharing between the $b$-quarks $z\left(p_{\mathrm{T}}\right)=$ $p_{\mathrm{T} \text {,subleading }} /\left(p_{\mathrm{T}, \text { leading }}+p_{\mathrm{T} \text {,subleading }}\right)$; and a quantity sensitive to the orientation of the gluon splitting relative to the gluon production plane $\Delta \theta_{p p g, g b b}$, which is the angle between the plane spanned by the beam line and the vector sum of the two track-jets, and the plane spanned by the two trackjets. The angular quantities are depicted in Figure 1. In addition to these quantities, the dimensionless mass $\log \left(m_{b b} / p_{\mathrm{T}}\right)$ is also measured, where the mass and $p_{\mathrm{T}}$ in the logarithm are computed from the four-vector sum of the two track-jets. The $b \bar{b}$ mass is an important observable for measurements and searches with Higgs and $Z$ bosons.
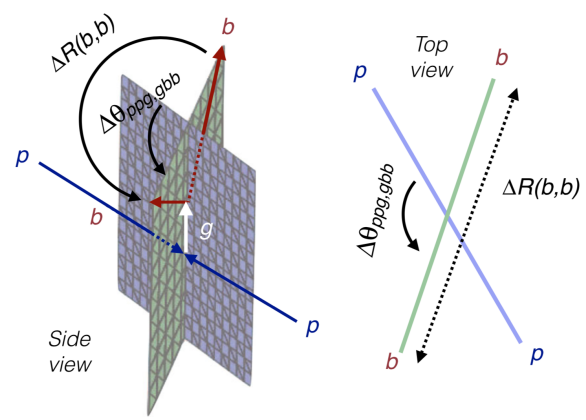

Figure 1: Schematic diagrams illustrating the $\Delta R(b, b)$ and $\Delta \theta_{p p g, g b b}$ observables. In this example the gluon is emitted at $\eta=0$ [4].

The offline analysis takes large-radius jets with $p_{\mathrm{T}}>450 \mathrm{GeV}$ and $|\eta|<2$ and requires the highest- $p_{\mathrm{T}}$ large radius jet to have at least two associated track-jets with $p_{\mathrm{T}}>10 \mathrm{GeV}$ and $|\eta|<2.5$. If there are more than two track-jets, only the leading two are used for subsequent analysis. The leading track-jet associated with the selected large-radius jet must be $b$-tagged. At particle level, events are required to have at least one large-radius jet with $p_{\mathrm{T}}>450 \mathrm{GeV}$. The leading jet needs to have at least two associated particle-level track-jets with $p_{\mathrm{T}}>10 \mathrm{GeV}$. Both of the associated small-radius jets must be identified as $b$-jets. This inclusive event selection produces a sample where QCD scattering processes dominate.

After the event selection, a flavour-fraction fit is used to remove contributions from processes other than $g \rightarrow b \bar{b}$. The distribution of the signed impact parameter significance $s_{d_{0}}$, computed for 
a given track as its transverse impact parameter with respect to the beam line $d_{0}$ divided by the uncertainty in $d_{0}$ from the track fit, is fitted to data using templates from simulation while letting the fraction of each flavor component float in the fit. Due to the long lifetime of $b$-hadrons, the values of $s_{d_{0}}$ for tracks in $b$-jets tend to be larger than those for tracks in c-jets and light-flavor jets. Therefore, the distribution of $s_{d_{0}}$ can be used to extract the flavor fractions using templates from simulation. For each track-jet, the $s_{d_{0}}$ from the track with the second largest $\left|s_{d_{0}}\right|$, called $s_{d_{0}}^{\text {sub }}$, is used for the extraction. Examples of the flavor-fraction determination fits are shown in Figure 2 for one bin of $\Delta R(b, b)$. The fitted fractions significantly disagree with the prefit Pythia predictions and suggest that further studies could improve the modeling of analyses sensitive to these fractions.
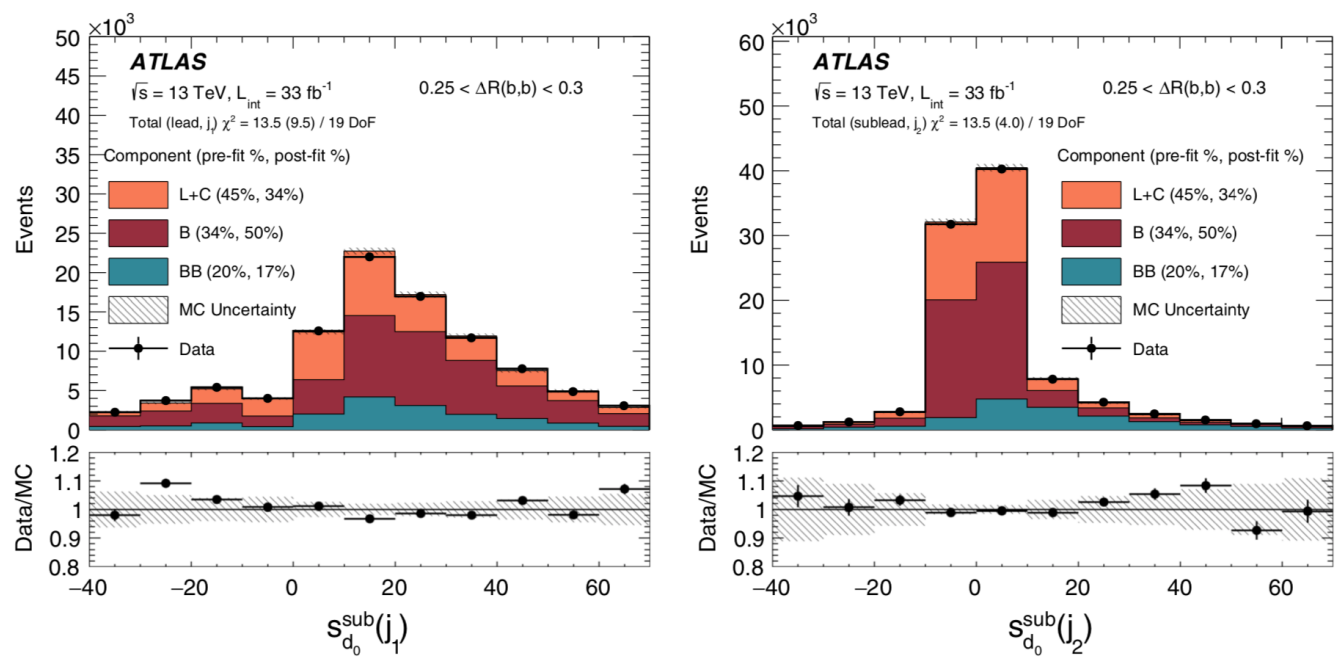

Figure 2: The distribution of $s_{d_{0}}^{\text {sub }}$ in data and in simulation, postfit, for the higher- $p_{\mathrm{T}}$ track jet (left) and for the lower- $p_{\mathrm{T}}$ track jet (right) in the bin $0.25<\Delta R(b, b)<0.3$. The three components are the signal double- $b$ (BB), the background single $b(\mathrm{~B})$, and the background non- $b$ components $(\mathrm{L}+\mathrm{C})$. Percentages reported in the legend indicate the prefit and postfit fractions of each component. Only data and MC statistical uncertainties are shown. The lower panel shows the ratio between data and the postfit simulation [4].

After subtracting the background from the detector-level distributions, the measured properties are unfolded to correct for the detector acceptance and resolution for direct comparison with particle-level models. The measurements are dominated by the theoretical modelling uncertainty. The large-radius jet energy scale dominates the experimental systematics.

The unfolded results along with multiple parton shower MC predictions are presented in Figure 3. The Sherpa predictions are generally more accurate than those from Pythia. There are significant differences between both generators and the data at low mass, low $z\left(p_{\mathrm{T}}\right)$ and for all $\Delta \theta_{p p g, g b b}$. This might point to a missmodelling of gluon polarization inside unpolarized hadrons.

\section{Properties of jet fragmentation using charged particles}

The details of the process that underlies the creation of net neutral hadrons from quarks and gluons with net QCD charge, fragmentation, is not fully understood. Jet formation is a complex multi-scale problem, including important contributions from QCD effects that cannot be described by perturbation theory. Measuring basic quantities related to fragmentation is therefore essential 

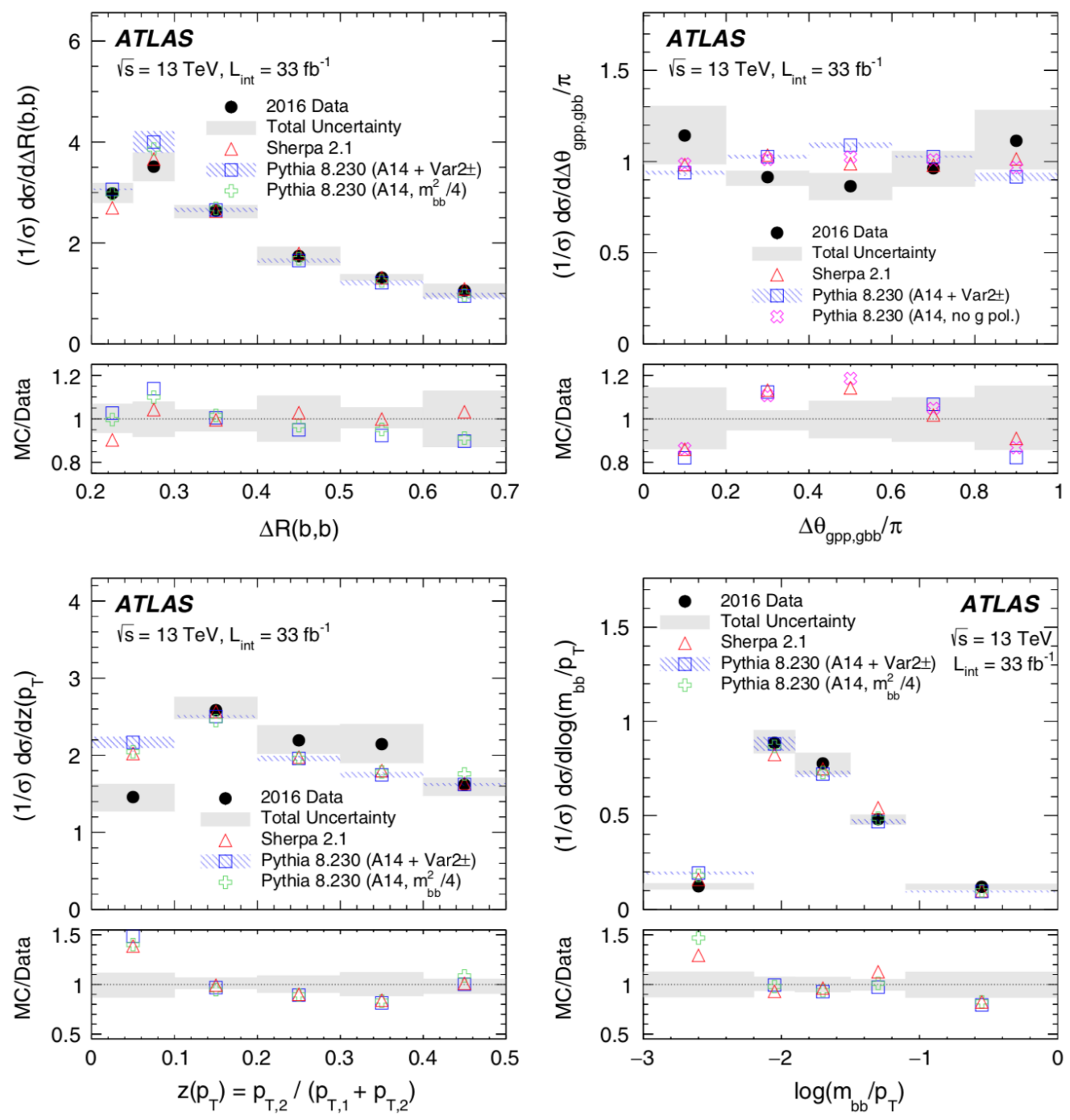

Figure 3: The unfolded distribution of $\Delta R(b, b)$ (top left), $\Delta \theta_{p p g, g b b}$ (top right), $z\left(p_{\mathrm{T}}\right)$ (bottom left), and $\log \left(m_{b b} / p_{\mathrm{T}}\right)$ (bottom right). Error bands represent the sum in quadrature of statistical and systematic uncertainties. These data are compared with predictions from the Pythia and Sherpa MC simulations [4].

to furthering our understanding of QCD. QCD-inspired models have free parameters that are tuned to data in order to best describe the radiation pattern inside jets [5]. This is in turn an important input to all analyses at the Large Hadron Collider (LHC) due to the ubiquity of jets. Measurements of jet shape and structure observables provide powerful constraints for future Monte Carlo event generator optimizations.

Quark- and gluon-initiated jets have different radiation patterns. As many analyses at the LHC target either quark-enriched or gluon-enriched processes, these radiation-pattern differences can be useful for jet tagging. Although the full radiation pattern inside jets is not calculable from first principles, the energy dependence of many observables can be calculated in perturbation theory.

The fundamental quantity is the fragmentation function, which describes the probability of finding a hadron $h$ with energy fraction $z$ of the parton $p$ that has energy $E$. A basic quantity related 
to the fragmentation function is the charged-particle multiplicity. Additional observables are also studied in order to probe the angular spread of jet fragmentation:

- Charged-particle multiplicity $\left(n_{c h}\right)$ : The number of charged particles inside a jet with $p_{\mathrm{T}}$ above some threshold.

- Summed fragmentation function: The distribution of the $p_{\mathrm{T}}$ fraction, $\zeta=p_{\mathrm{T}}^{\text {particle }} / p_{\mathrm{T}}^{\text {jet }}$, is studied inside jets. The measured quantity is $F(\zeta)=\left(1 / N^{\text {jet }}\right) d n_{c h} / d \zeta$. In addition to this, summary statistics of the $F(\zeta)$ distribution are extracted to show how the distribution evolves with $p_{\mathrm{T}}^{\text {jet }}$.

- Relative transverse momentum: $p_{\mathrm{T}, \mathrm{rel}}=p_{\mathrm{T} \text {,chargedparticle }} \times \sin \Delta \phi$, where $\Delta \phi$ is the angle between the momentum vector of the constituent charged particle and the jet axis in the transverse plane.

- Radial profile: The number of charged particles in various annuli around the jet axis.

The ATLAS collaboration has recently measured properties of jet fragmentation using chargedparticle tracks [6]. The measurement presented there represents a significant extension of previous work. The accessible jet energy range is increased to probe the substructure of jets with $p_{\mathrm{T}}$ up to 2.5 TeV. The precision of the measurement has improved due to advances in track reconstruction inside jets, including the use of the additional insertable B-layer detector and new algorithms for tracking inside dense environments. These new data therefore probe broader and deeper aspects of the radiation pattern inside jets across an extended phase space.

In this study both particle-level and detector-level events are required to have at least two jets with $|\eta|<2.1$ and the leading two such jets must satisfy $p_{\mathrm{T}, \text { lead }} / p_{\mathrm{T} \text {,sublead }}<1.5$.

In order to expose differences between quark and gluon jets, the more forward and more central of the two jets are distinguished and measured separately. For a fixed jet $p_{\mathrm{T}}$, higher- $|\eta|$ jets are more often quark-initiated due to valence quarks scattering off gluons. For a fixed $|\eta|$, the quark fraction increases with jet $p_{\mathrm{T}}$ due to the relative increase in valence-quark scattering off a quark or gluon compared with gluon-gluon scattering. The unfolded averages of the measured observables are presented as a function of the jet $p_{\mathrm{T}}$ in Figure 4.

The more forward and the more central of the two selected jets can be separated to study differences between the radiation patterns within quark and gluon jets. Using the fraction of quark jets in the two jet samples, one can extract the quark and gluon jet fragmentation properties separately. A key challenge with the extraction is that it strongly depends on simulation. A new approach that does not require the input of any fractions is topic modeling [7]. The extracted topics using $n_{c h}$ are shown in the left panel of Figure 5. The first topic is well aligned with quarks and the second topic is more gluon-like. This alignment is better for quarks than for gluons, but the second topic does converge to the gluon distribution at high jet $p_{\mathrm{T}}$. The right panel of Figure 5 shows $\left\langle n_{c h}\right\rangle$ as a function of $p_{\mathrm{T}}^{\text {jet }}$ for both extracted quark-like and gluon-like jets as well as the topic extraction along with the prediction for the pure quark/gluon case. Gluon jets from data deviate significantly from simulation and from the calculation at high jet $p_{\mathrm{T}}$; this is also true to a lesser extent for quark jets, which seem to have a different slope that is most prominent at low jet $p_{\mathrm{T}}$. 

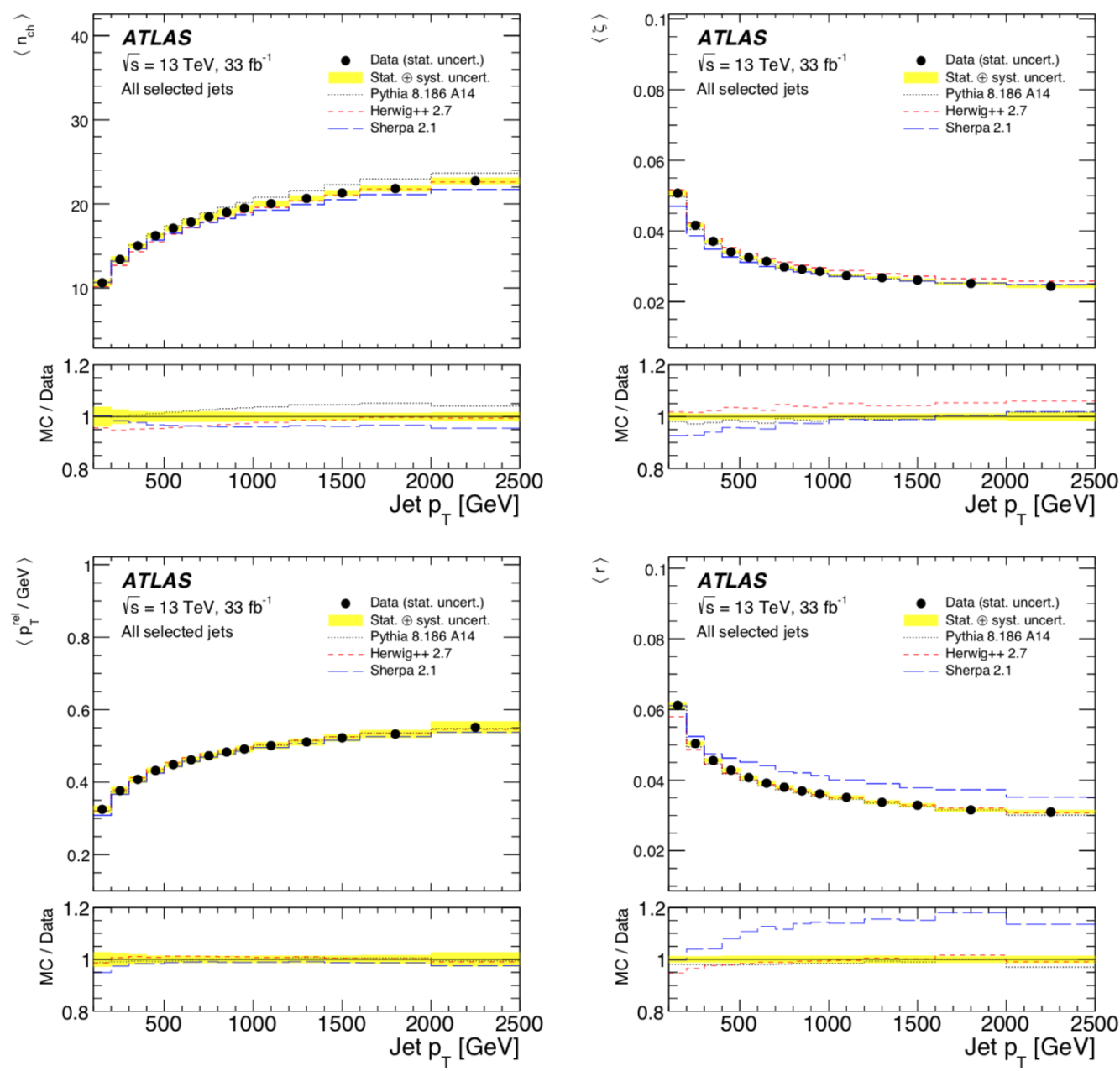

Figure 4: The average values of the unfolded distributions of (top left) charged-particle multiplicity $n_{c h}$,

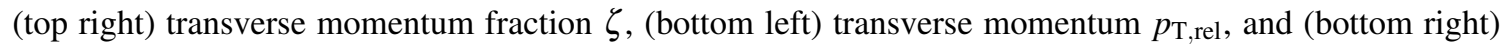
radial profile as a function of the jet transverse momentum $p_{\mathrm{T}}$. The lower panel shows the ratio of various MC predictions to the data, with the total uncertainty band centered on the data at unity [6].

\section{Summary}

This document summarises recent measurements related to jet fragmentation at $13 \mathrm{TeV}$ using a dataset corresponding to an integrated luminosity of $33 \mathrm{fb}^{-1}$ recorded by the ATLAS detector at the LHC.

In the measurement of various properties of $g \rightarrow b \bar{b}$ at high $p_{\mathrm{T}}$ and low $\Delta R(b, b)$ a flavorfraction fit is used to remove contributions from processes other than $g \rightarrow b \bar{b}$. The fitted fractions significantly disagree with the prefit Pythia predictions and suggest that further studies could improve the modeling of analyses sensitive to these fractions. The measured quantities are compared at the particle level between the distributions and various models of jet formation. Simulations from the Sherpa event generator generally provide a better model than Pythia, especially for the $\Delta \theta_{p p g, g b b}$ observable, which is sensitive to the modeling of the gluon polarization.

Multiple jet properties, including the charged-particle multiplicity, the momentum fraction 

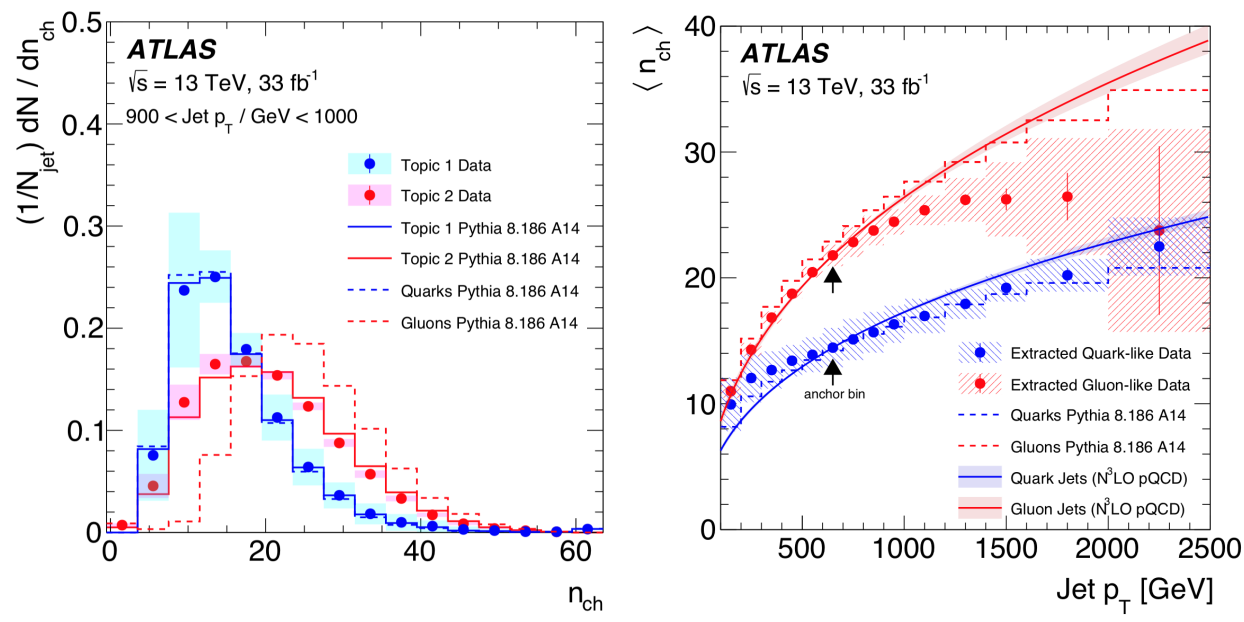

Figure 5: (Left) The jet topics extracted using charged-particle multiplicity $n_{c h}$ together with the topics and quark and gluon distributions from Pythia. (Right) The dependence on jet transverse momentum $p_{\mathrm{T}}$ of the mean charged-particle multiplicity $\left\langle n_{c h}\right\rangle$ for quark and gluon jets in data and in Pythia as well as from a calculation using perturbative QCD. The uncertainty bands on the data include both statistical and systematic uncertainties while the error bars are due to statistical uncertainties only [6].

carried by charged particles, and angular properties of the radiation pattern inside jets are studied in the measurement of track-based jet fragmentation functions. There are key areas where there are significant disagreements between the ATLAS default MC simulation and the data. The radial profile is systematically broader in data than in simulation, but the momentum transverse to the jet axis and the momentum fraction are well modeled within the precision of this measurement. Near $1 \mathrm{TeV}$ in jet $p_{\mathrm{T}}$, these measurements have achieved percent-level uncertainties for a variety of observables. In addition to measuring the forward, central, and combined jet distributions, the forward and central jet spectra are considered separately to study quark- and gluon-like distributions. A first measurement of topic modeling for the charged-particle multiplicity provides a promising alternative to traditional methods of extracting quark- and gluon-jet distributions that use input from simulation. The simulations provide a reasonable description of the quark-like data across the jet $p_{\mathrm{T}}$ range presented in this measurement, but the gluon-like data have systematically fewer charged particles than the simulations.

\section{References}

[1] ATLAS collaboration, Phys. Lett. B 779, 24 (2018). CMS collaboration, Phys. Rev. Lett. 120, 071802 (2018).

[2] ATLAS collaboration, Phys. Lett. B 774, 494 (2017), Phys. Lett. B 786, 59 (2018).

[3] ATLAS collaboration, 2008 JINST 3 S08003.

[4] ATLAS collaboration, Phys. Rev. D 99, 052004 (2019).

[5] A. Buckley et al., Phys. Rep. 504 (2011) 145.

[6] ATLAS collaboration, Phys. Rev. D 100, 052011 (2019).

[7] E. M. Metodiev and J. Thaler, Phys. Rev. Lett. 120, 241602 (2018). 